\title{
géologie et génie civil des ouvrages souterrains sous la chaîne du Chat et de l'Épine (Savoie) *
}

\author{
par \\ Jean Darcy \\ Ingénieur géologue \\ Centre d'Études des Tunnels \\ Ministère de l'Équipement
}

\section{Avant-propos}

Depuis une quinzaine d'années, les géologues et les spécialistes des travaux souterrains ont eu à étudier différents projets et à en assurer la réalisation dans la chaîne des Monts du Chat et de l'Épine, en Savoie.

II paraissait intéressant de faire la synthèse des enseignements recueillis lors de ces travaux, tant sur le plan de la Géologie que sur celui du Génie Civil.

En effet, l'historique des prévisions des structures géologiques, l'hydrogéologie du massif, et le comportement des roches rencontrées méritent une réflexion générale sur le double problème de l'étude des projets et de la conduite des travaux souterrains dans le cas précis des chaînons jurassiens, et peut-être dans le cas plus général des structures géologiques profondes et complexes.

\section{La chaîne des Monts du Chat et de l'Épine}

\subsection{Géographie et morphologie (fig. 1)}

Les Monts du Chat et de l'Épine forment une chaine continue d'orientation Nord-Sud qui domine la dépression de Chambéry et du Lac du Bourget située immédiatement à l'Est.

Ce relief présente une ligne de crête d'altitude régulièrement voisine de $1000 \mathrm{~m}$ et culmine à $1400 \mathrm{~m}$ environ. II s'agit donc d'un obstacle géographique important que les ouvrages modernes (routes, voies ferrées, émissaires) doivent franchir en galerie.

\subsection{Géologie}

La chaîne du Chat et de l'Épine est le chaînon jurassien le plus oriental à cette latitude, puisqu'il est situé en bordure du sillon subalpin constitué par la dépression de Chambéry et du Lac du Bourget. II apparaît sur la carte géologique comme un anticlinal de Jurassique supérieur et de Crétacé

• Comité Français de Géologie de l'Ingénieur. Séance du 9 février 1978. dont les flancs s'ennoient sous les formations molassiques miocènes. Un accident tectonique frontal affecte la bordure Ouest de ce chainon sur toute sa longueur.

L'observation de surface est relativement facile sur le flanc Est de structure régulière ; par contre, elle est difficile sur le flanc Ouest souvent abrupt et recouvert de formations quaternaires - dépôts glaciaires ou éboulis - abondantes qui masquent souvent l'accident frontal précité.

\section{Les principaux ouvrages souterrains}

Du Nord au Sud, trois ouvrages ont fait l'objet de travaux récemment, ou sont en cours d'exécution. Les principales caractéristiques de ces ouvrages, localisés sur la figure 1 , sont résumées dans le tableau 1, ci-après:

\begin{tabular}{l|c|c|c|c|c}
\hline & $\begin{array}{c}\text { Date de } \\
\text { construction }\end{array}$ & $\begin{array}{c}\text { Longueur } \\
(\mathrm{m})\end{array}$ & $\begin{array}{l}\text { Cote } \\
\text { NGF }\end{array}$ & $\begin{array}{c}\text { Couver- } \\
\text { ture (m) })\end{array}$ & $\begin{array}{l}\text { Section } \\
\left(\mathrm{m}^{2}\right)\end{array}$ \\
\hline $\begin{array}{l}\text { Tunnel routier } \\
\text { du Chat }\end{array}$ & $1929 \cdot 1931$ & 1486 & 400 & 490 & 51 \\
\hline $\begin{array}{l}\text { Galerie du } \\
\text { Bourget }\end{array}$ & $\begin{array}{c}\text { en cours } \\
\text { (depuis 1974) }\end{array}$ & $12325^{*}$ & 225 & 1300 & 7 \\
\hline $\begin{array}{l}\text { Tunnel } \\
\text { autoroutier } \\
\text { de l'Epine }\end{array}$ & & & & & \\
$\begin{array}{l}\text { galerie de } \\
\text { reconnaissance }\end{array}$ & $1967-1968$ & 3120 & 400 & 600 & 7 \\
-tunnel & $1972-1974$ & 3120 & 405 & 600 & 95 \\
\hline
\end{tabular}

* Longueur totale du tronçon en souterrain. La partie en souterrain sous les Monts du Chat est longue de $5500 \mathrm{~m}$.

Le tunnel routier du Chat a fait l'objet d'une réfection complète en 1972 et 1973. A ce titre, il a été examiné en détail et des observations ont pu être effectuées sur le comportement des roches et l'hydrogéologie.

La galerie de reconnaissance du tunnel de l'Épine et le tunnel autoroutier de l'Épine (Autoroute A-43 Lyon-Chambéry) ont fait l'objet d'études depuis 1963 et leur exécution a pu être suivie dans le détail. 


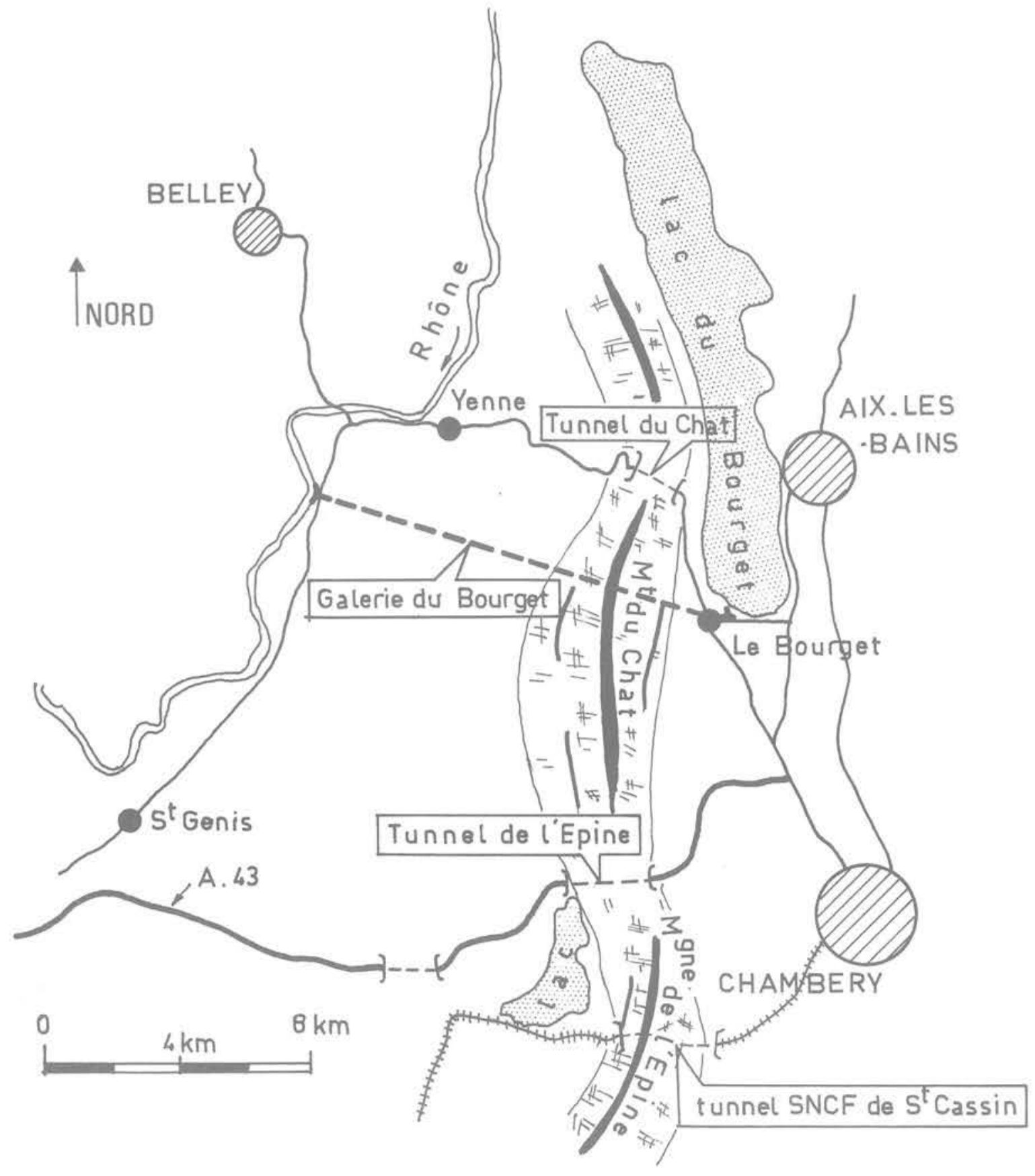

Fig. 1 Situation des ouvrages souterrains 
La galerie du Bourget est destinée à évacuer vers le Rhône les eaux usées, après épuration, des agglomérations de Chambéry et d'Aix-les-Bains; cette galerie servira également et éventuellement à assainir le lac du Bourget en évacuant les eaux profondes polluées. Cette galerie en cours d'exécution présente un grand intérêt en ce qui concerne les problèmes d'interprétation structurale qu'elle a posés, et le rôle du Géologue dans les travaux souterrains. Pour mémoire, il faut citer le tunnel ferroviaire de Saint-Cassi qui n'a pas fait l'objet de travaux récents dont nous ayons eu connaissance.

\section{Structure géologique au droit des ouvrages souterrains}

\subsection{La stratigraphie des formations rencontrées}

Les roches rencontrées dans les différents ouvrages vont du Bajocien à l'Helvétien supérieur, exception faite des formations quaternaires qui ne feront pas l'objet de développements particuliers dans cet exposé. Une description lithologique sommaire en est donnée ci-après :

- Jurassique moyen

Bajocien (Baj) calcaires à entroques, spathiques ou siliceux

Bathonien (Bat) marno-calcaires à la base et calcaires à silex

- Jurassique supérieur

Callovien (Cal)

Oxfordien (Oxf) Kimmeridgien (K)

caicaire à oolithes ferrugineuses (niveau repère de faible épaisseur) marnes et marno-calcaires

à la base - calcaires en gros bancs, puis calcaires en petits bancs décimétriques bien lités dans la partie moyenne et au sommet - calcaires massifs ou récifaux

présence générale d'une zone dolomitisée

Portlandien ( $P$ ) calcaires massifs à la base, bien stratifiés au sommet

Purbeckien (Purb) calcaires lacustres à niveaux bréchiques et intercalations de marnes à galets, indurées

- Crétacé

Valanginien (V)

calcaires massifs dans l'ensemble. spathiques, ou à faciès subrécifal. Tendance marno-calcaire ou marneuse au sommet

Hauterivien $(\mathrm{H})$ calcaires siliceux, calcaires argileux et marnes

Barrémien (B) calcaires et marno-calcaires jaunes et gros bancs à faciès urgonien

\section{- Oligocène}

Chattien (ch)

conglomérat et marnes rouges

- Miocène

Burdigalien (Burd) conglomérat et grès verts

Helvétien (Helv) marnes et grès à la base

ensemble argilo-silto-gréseux dans la partie médiane

sables peu cimentés intercalés de bancs de grès au sommet.

\subsection{Le tunnel du Chat - structure géologique}

La coupe géologique des Monts du Chat à l'aplomb du tunnel s'est trouvée parfaitement vérifiée lors du creusement du souterrain. En effet, l'extrapolation des observations de surface très faciles a permis d'établir une coupe géologique prévisionnelle précise car la structure était simple: I'anticlinal s'est déversé vers l'Ouest et cisaillé au droit de l'accident frontal qui met en contact le Valanginien et le Bajocien (fig. 2), II en est résulté une structure simple qui règne en profondeur au moins jusqu'à la cote du tunnel routier.

Les seuls anomalies de la structure sont les plissotements nombreux observés dans l'Oxfordien et le Kimmeridgien inférieur: le comportement de ces roches bien litées et moins résistantes que les formations voisines est bien connu et général.

\subsection{Le tunnel de l'Épine - reconnaissances et structure géologique}

Les premières reconnaissances de surface ont montré que la Montagne de l'Épine présentait, au moins extérieurement, l'aspect d'un anticlinal légèrement assymétrique à flanc Ouest redressé. L'accident frontal à l'Ouest est visible, mais difficilement interprétable.

Ces constatations ont conduit à dessiner la coupe de la figure 3 , c'est-à-dire un anticlinal régulier dont le cœur serait constitué de Jurassique moyen. Cependant, étant donné la tendance bien connue de l'Oxfordien et du Kimmeridgien inférieur à se plissoter et à engendrer des surépaisseurs notables, il a paru nécessaire de s'assurer de la validité de cette coupe par un sondage profond de $637 \mathrm{~m}$ qui a traversé I'anticlinal jusqu'à la cote du projet. Ce sondage a été partiellement carotté et le forage a fait l'objet de plusieurs diagraphies - logs électriques, soniques, pendagemétrie. En outre, la rencontre de plusieurs zones karstiques a engendré des difficultés - pertes de fluide d'injection, nécessité de cimentations. Seul le Kimmeridgien inférieur a été rencontré ; il présente son faciès habituel de calcaires sombres en petits bancs à l'exception d'une zone dolomitisée de $120 \mathrm{~m}$ d'épaisseur environ située a $200 \mathrm{~m}$ de profondeur. La pendagemétrie indiquait des discontinuités d'orientation très variable. La structure prévisionnelle a donc dû être corrigée compte tenu des plissements annexes du Kimmeridgien inférieur. Une galerie de reconnaissance a été ensuite exécutée pour servir également de galerie de drainage, compte tenu de la forte probabilité de rencontre de venues d'eau nombreuses et importantes. Cette galerie a été implantée entre les deux tunnels autoroutiers prévus, et à $5 \mathrm{~m}$ en dessous. Elle a confirmé et précisé les prévisions déjà affinées par le sondage, et la coupe géologique définitive a pu être établie (fig, 4).

II a été possible de mettre en évidence les trois zones de contraintes conformes au schéma théorique relatif aux plis anticlinaux.

- zones en extension à l'Est et à l'Ouest avec fissuration ouverte à remplissage d'argile ou de calcite.

- zone neutre correspondant approximativement à la zone dolomitisée.

- zone centrale en compression caractérisée par des plissotements et plusieurs plis secondaires dans le Kimmeridgien inférieur.

II faut signaler que cette galerie a fait l'objet de nombreux essais de mécanique des roches, parmi lesquels des mesures de contraintes par M. Panet [5] et des essais de prédécoupage par $P$. Weber [5] qui a bien mis en évidence l'influence des contraintes initiales dans la roche sur le phénomène de propagation de la fissure. Les résultats de mesures de contraintes se sont révélés très dispersés, notamment dans la zone centrale où l'on a observé des contraintes très élevées, mais également des tractions, ce qui s'explique par la présence de nombreux plis secondaires qui présentent chacun des zones en compression et des zones en tension. 


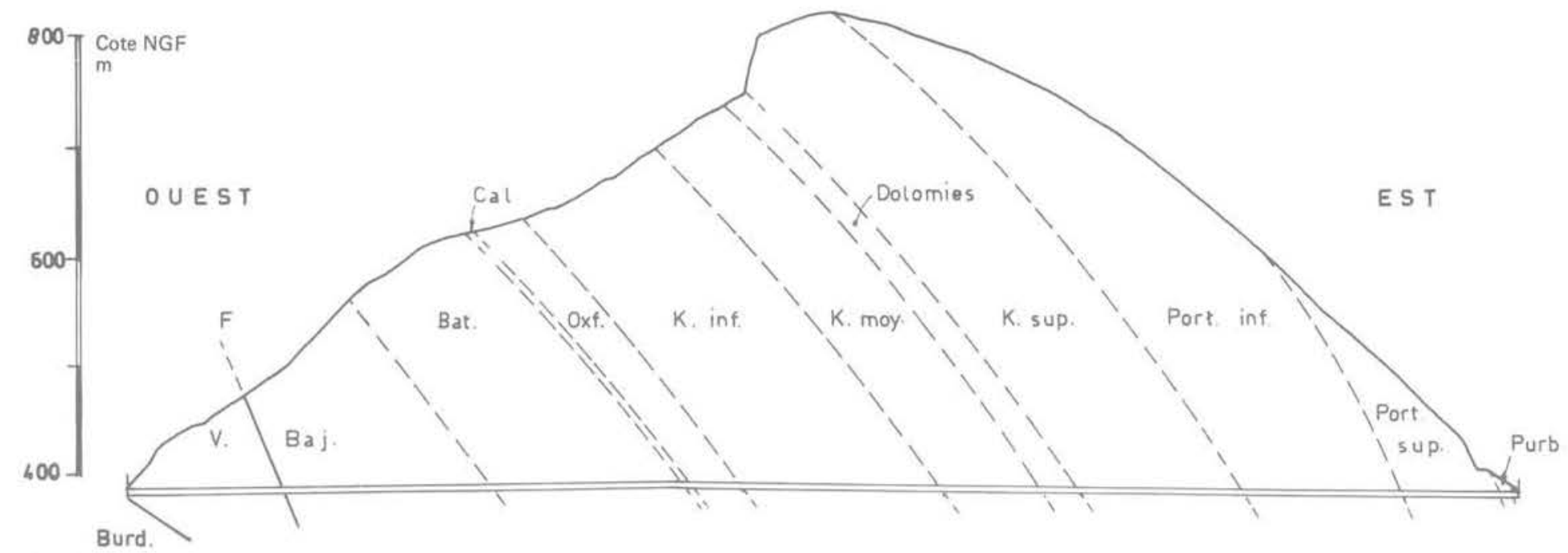

P.C 350.00

1500

1000

500

0

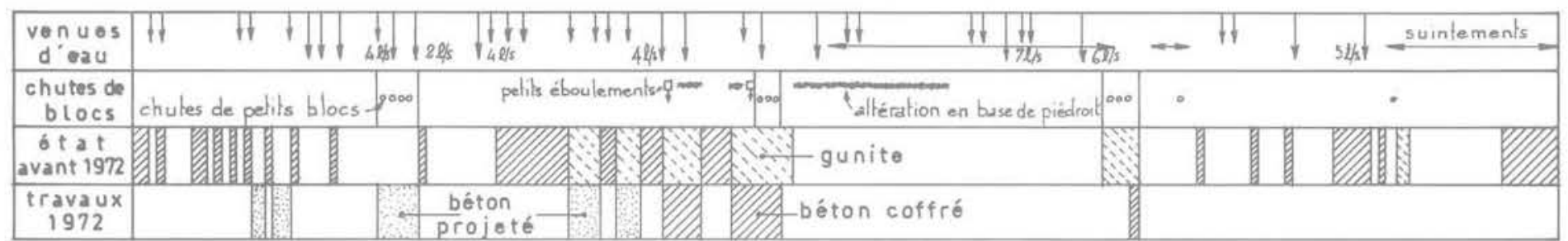

Fig. 2 Tunnel du Chat, Géologie. Venues d'eau. Revêtement

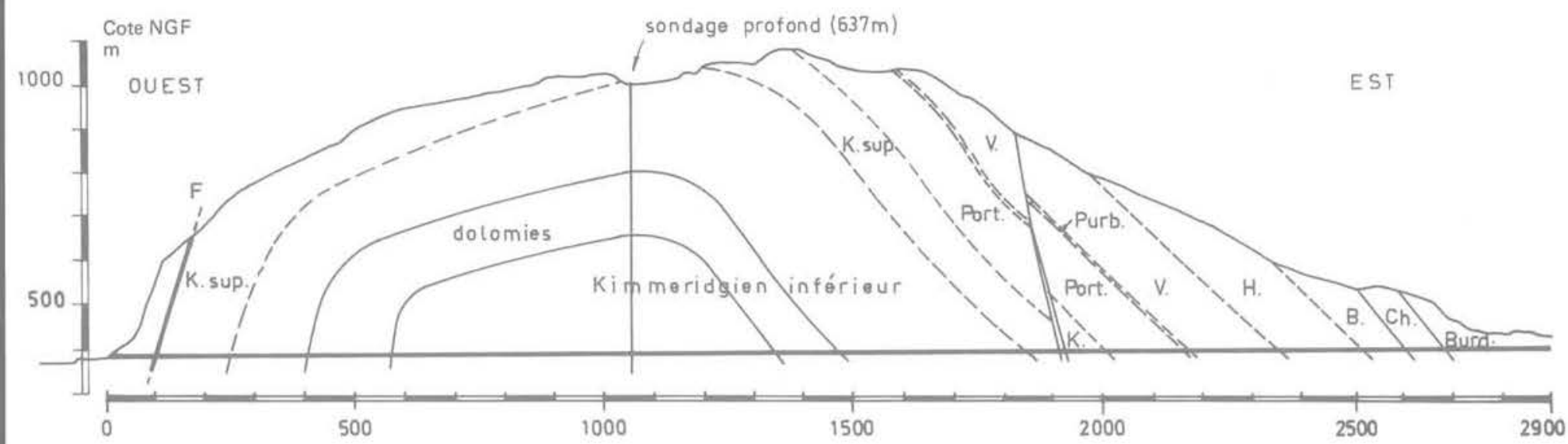

\begin{tabular}{|c|c|c|c|c|c|c|c|}
\hline $\begin{array}{l}\text { venues } \\
\text { d'edu }\end{array}$ & 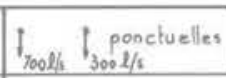 & diffuses & ponct. diff & par d'eau & \begin{tabular}{|c|} 
diffusea \\
ponctuelles
\end{tabular} & t800 $2 / 5$ ponctuelles & $\begin{array}{l}\text { diffuses } \\
\text { II poncluetles }\end{array}$ \\
\hline kerats & & & & & $501 / \mathrm{mo}$ & & \\
\hline décompression & & & & . zône o & mpression & & $\begin{array}{l}\text { dérollement } \\
\text { de plaques } \\
\text { dins molasse }\end{array}$ \\
\hline $\begin{array}{c}\text { soulénement } \\
\text { cintrifres }\end{array}$ & & & & & & & \\
\hline
\end{tabular}

Fig. 3 Tunnel de l'Epine. Coupe géologique prévisionnelle 


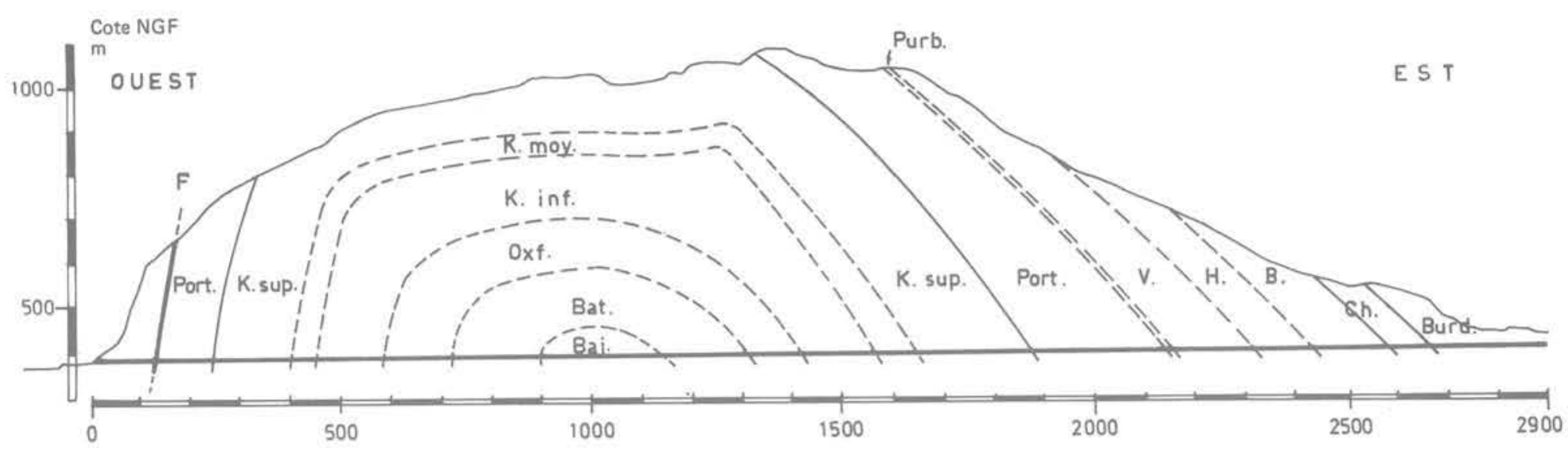

Fig. 4 Galerie de reconnaissance du tunnel de l'Epine. Géologie. Hydrogéologie. Soutènement

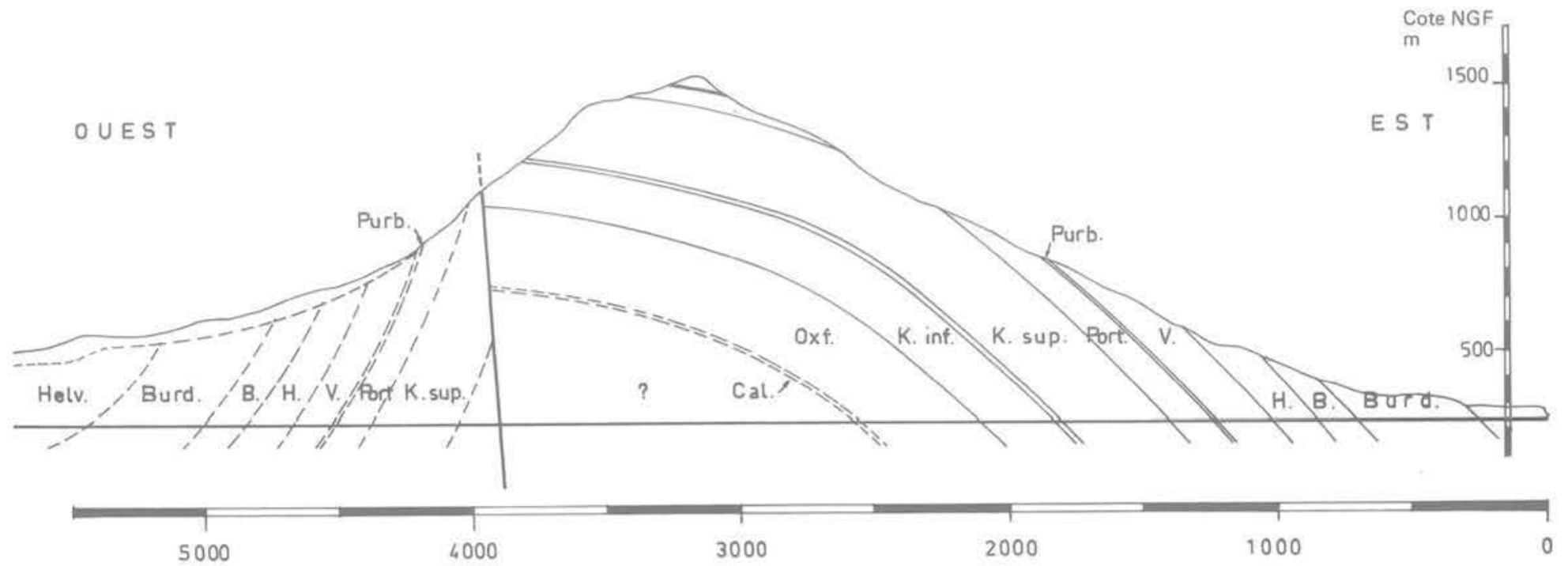

Fig. 5 Galerie du Bourget. Coupe géologique prévisionnelle effectuée en 1976

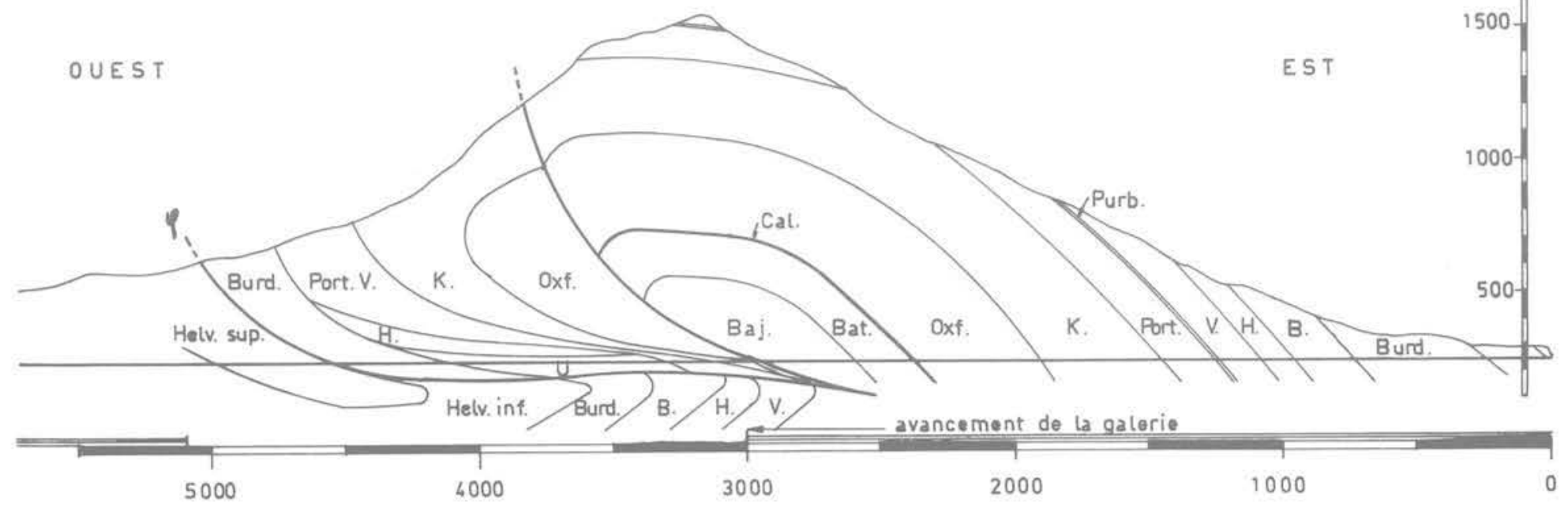

Fig. 6 Galerie du Bourget. Coupe prévisionnelle établie à la fin de 1977 


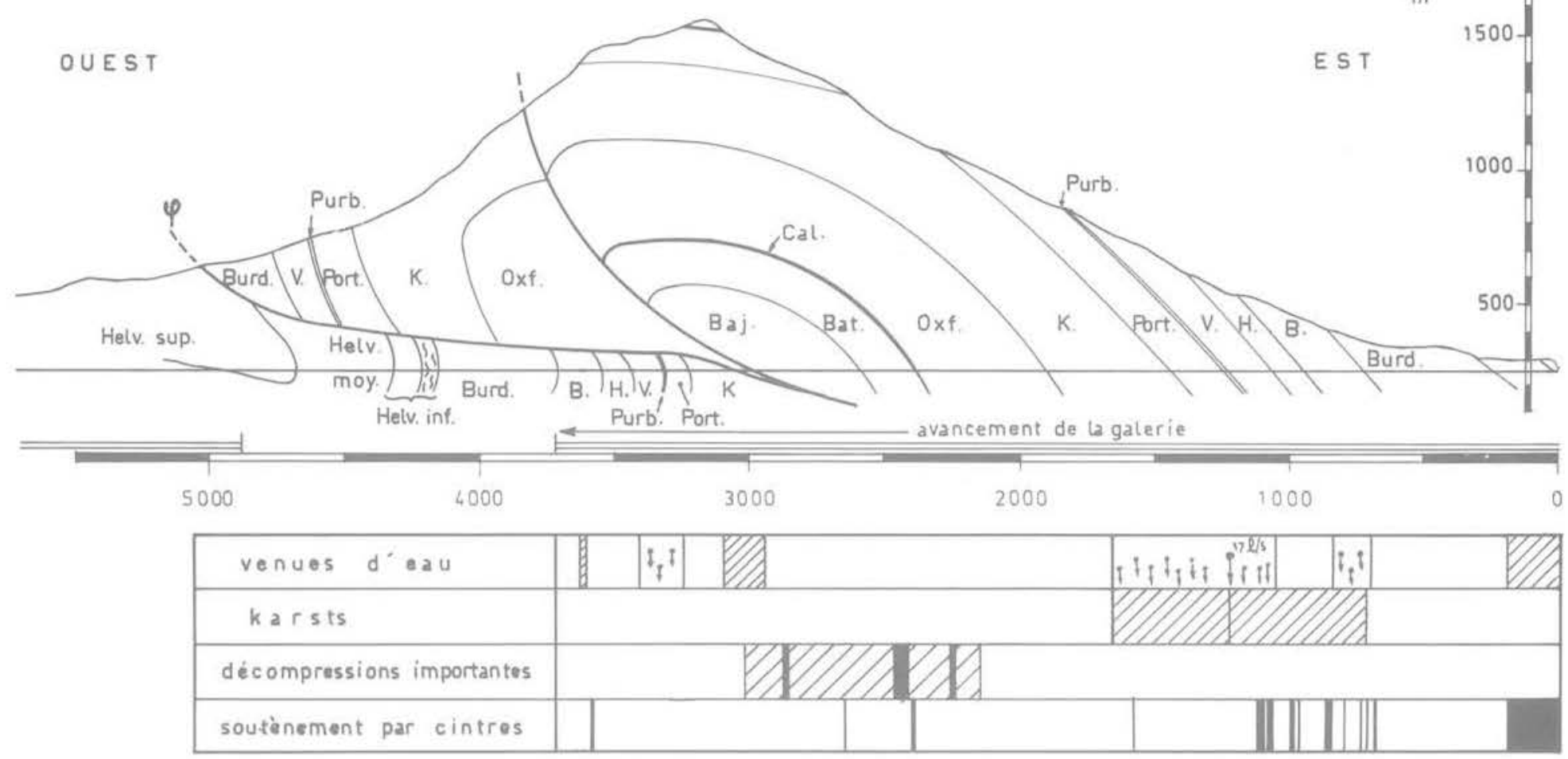

Fig. 7 Galerie du Bourget. Coupe géologique en janvier 1978. Hydrogéologie. Génie civil

\subsection{La Galerie du Bourget - Structure géologique}

La Galerie du Bourget est située entre les tunnels du Chat et de l'Épine, à $4 \mathrm{~km}$ au Sud de celui-ci et à $9 \mathrm{~km}$ au Nord de cette dernière.

L'étude géologique du projet a été effectuée et fondée essentiellement sur la synthèse des connaissances apportées par les deux ouvrages précédents, synthèse complétée par un lever de surface.

La coupe géologique prévisionnelle (fig. 5) montre à l'Est un flanc d'anticlinal régulier analogue à celui des tunnels du Chat et de l'Épine. Les auteurs ont limité leurs prévisions au Kimmeridgien, faisant bien apparaître l'incertitude qui régnait dans la partie centrale, au droit de l'Oxfordien et de la zone comprimée. Ceci montrait bien qu'il y avait des risques avec répercussion sur le montant prévisionnel de la dépense.

Sur le flanc Ouest, l'accident tectonique affecte le Kimmeridgien supérieur, puis toute la série supérieure est masquée par les formations quaternaires, d'où l'impossibilité de localiser la position des différents étages avec précision. Le dernier étage de calcaire prévu est le Barrémien qui s'ennoie sous la molasse burdigalienne tant à l'Est qu'à l'Ouest.

Les travaux de ce troncon ont commencé à l'Est en attaque descendante. La coupe prévisionnelle a été vérifiée avec une bonne précision jusqu au Callovien atteint au milieu de 1977, après traversée des plissements secondaires attenduus dans l'Oxfordien et le Kimmeridgien inférieur. Maitre d'œuvre et Entrepreneur étaient alors dans l'incertitude en ce qui concernait la nature des terrains à venir, leur comportement au droit de l'accident tectonique, et les venues d'eau. Une concertation permanente a alors été instaurée entre les Géologues Conseils du Maître d'œuvre, de l'Entrepreneur, et ceux de I'Université.
Toutes les hypothèses possibles de structure ont été passées en revue, et notamment ont été envisagées, les possibilités suivantes :

- pendage moins accentué de l'accident dessiné, d'où éventualité de le rencontrer plus tôt.

- existence d'un grand chevauchement à faible pendage : cette hypothèse résulte de l'interprétation d'un sondage pétrolier de 1975 dans le massif de la Chambotte qui a mis en évidence une telle structure dans un autre chainon jurassien. D'autres structures analogues ont d'ailleurs souvent été rencontrées dans le Jura plus au Nord. On pouvait d'ailleurs imaginer que ce chevauchement intéresse la molasse, à la suite de travaux récents et non encore publiés sur les formations miocènes de la région et qui faisaient mention de surépaisseurs anormales du Miocène.

Les coupes des figures 6 et 7 ont alors été dessinées. Elles montrent l'éventualité d'une structure plus complexe que celle initialement prévue ainsi que la possibilité de rencontrer les molasses miocènes aux environs du point métrique 3500 , fait très important pour l'économie et les délais du chantier car l'avancement $y$ est beaucoup plus lent et un soutènement plus lourd est nécessaire dans les grès silteux et argileux.

Parallèlement à cette démarche, il a été mis en place sur le chantier, tout un dispositif d'alerte de manière à pouvoir organiser à tout moment la concertation entre les divers géologues concernés. Le personnel du chantier a été averti d'avoir à examiner en permanence le terrain du front de taille et avertir les géologues de toute variation brutale ou progressive du pendage, de l'apparition d'une fissuration plus intense ou de diaclases remplies de calcite, des variations de débit, température et résistivité de l'eau et aussi, bien sûr, de tout changement de nature lithologique de la roche. 
C'est finalement une succession de terrains et une tectonique cohérentes avec l'hypothèse de la figure 7 qui ont été rencontrées. De nombreux petits accidents cassants peu inclinés ont été observés dès le point métrique 3000 et dès que la série a été bien recalée par étude de la microfaune et des microfaciès, la coupe a pu être précisée et le Maître d'œuvre averti de la haute probabilité de rencontrer de la molasse aux environs du point métrique 3700 .

Sur le plan de la mécanique des roches, la partie du massif située à l'Est des grands accidents tectoniques présente des caractéristiques analogues à la Montagne de l'Épine au droit du tunnel autoroutier. On retrouve bien une zone centrale comprimée qui se développe vers l'Est jusqu'à I'Oxfordien et au Kimmeridgien inférieur plissoté et une zone en extension avec fissures ouvertes et développement de karsts. La zone neutre semble très réduite.

\section{Quelques aspects du génie civil liés à la nature et au comportement des roches}

\subsection{Le Tunnel du Chat}

Ce tunnel routier s'est avéré d'un niveau de confort insuffisant eu égard du trafic de l'itinéraire aux environs de 1970. Une réfection complète a alors été décidée sous le double aspect de la ventilation et de l'éclairage, et du génie civil. En ce qui concerne le génie civil, il convenait de remédier aux nombreuses venues d'eau et à des chutes de pierres, l'ouvrage n'étant que très partiellement revêtu, et d'assainir la chaussée dont le drainage s'avérait insuffisant. Les travaux exécutés sont schématisés sur la figure 8 . 11 a été réalisé :

- des anneaux de béton soit coffré soit projeté dans les zones instables ou altérables.

- un approfondissement des caniveaux et des drains,

- un chemisage complet de l'ouvrage par tôles parapluie.

Préalablement à ces travaux, un examen complet de l'ouvrage et de I'historique de son percement et de son exploitation a été effectué. II s'agissait en effet de bien connaître le tunnel et de mettre en place, dans un souci de sécurité évident, tous les revêtements indispensables à la pérennité de l'ouvrage, et en tenant compte du fait que lexamen du gros œuvre et des sections non revêtues ne pourrait plus être que difficile et partiel après la pose des tôles parapluie.

La stabilité des roches s'est avérée assez bonne lors de l'exécution de l'ouvrage. Les principales instabilités qui sont apparues dans le temps étaient liées à des zones fissurées et le plus souvent aquifères, à proximité de la faille par exemple, au droit des karsts dans la partie Est, et dans leszones de marnes ou de calcaires argileux plissotés où l'altération a joué un rôle prépondérant.

Les venues d'eau sont réparties sur toute la longueur du tunnel, ce qui est normal dans une telle structure où les écoulements se font parallèlement à la stratification. Les principales venues d'eau sont cependant concentrées aux têtes, dans les zones de faible couverture, et à fissuration ouverte et au droit de l'accident tectonique. Les débits sont faibles dans la zone centrale, dans le Kimmeridgien inférieur et l'Oxfordien comprimés et relativement imperméables de par leur nature lithologique. Les débits cumulés sont de $25 \mathrm{l} / \mathrm{s}$ pour chaque moitié du tunnel.

Ce débit relativement faible s'explique par le fait de la position élevée du tunnel dans la chaîne à 400 m NGF par rapport au réseau hydrographique au pied des versants : $200 \mathrm{~m}$ NGF pour le Lac du Bourget à l'Est et $300 \mathrm{~m}$ NGF au Lac du Chevelu à l'Ouest. Le tunnel ne joue donc pas un rôle véritable de drain: il ne fait qu'intercepter quelques circulations d'eau. Une modification des propriétés hydrauliques du massif s'est produite depuis le percement; les exploitants ont en effet constaté que l'arrivée de l'eau dans le tunnel à la suite de fortes précipitations se produisait maintenant avec un décalage de 0,5 jour contre 2 jours au moment de la construction. Le lessivage de fissures initia- lement colmatées a donc probablement eu lieu, ce qui accroît la vitesse de percolation de l'eau dans le massif. Des phénomènes de dissolution sont probables également. Des karsts, colmatés pour la plupart, ont été rencontrés aux extrémités du tunnel, notamment dans le Portlandien à I'Est.

Les revêtements mis en place avant 1972 avaient été motivés pour la plupart par l'abondance des venues d'eau. Une étanchéité relative avait été obtenue par canalisation de ces venues d'eau, puis mise en place de béton coffré, ou gunitage sur un grillage.

La détermination des zones à revêtir avant mise en place des tôles parapluie a été faite a partir d'un examen détaillé de la voûte complété par une auscultation dynamique dans les zones douteuses. Cette auscultation a été effectuée par dispositifs microsismiques comprenant 12 capteurs espacés de $2 \mathrm{~m}$ avec tirs en bout de ligne et tirs lointains. Ces dispositifs ont été complétés par carottages sismiques de $4 \mathrm{~m}$ de longueur où ont été effectuées des mesures de vitesses par tranche de $1 \mathrm{~m}$. Ces mesures ont permis de déterminer la vitesse longitudinale de la zone décomprimée et du rocher sain, l'épaisseur de la zone décomprimée, le module dynamique du rocher sain, et le "délai» à chaque capteur, lequel est fonction de l'épaisseur et de la vitesse de la zone décomprimée. Les zones de risque ont pu être ainsi classées et leur revêtement par béton coffré ou projeté a pu être décidé. Le tableau 2 indique quelques résultats caractéristiques.

Zone décomprimée

\begin{tabular}{|c|c|c|c|}
\hline & \multirow{2}{*}{$\begin{array}{c}E_{\text {dyn }} \\
\text { (MPa) }\end{array}$} & \multirow[b]{2}{*}{ vitesse $(\mathrm{m} / \mathrm{s})$} & \multirow[b]{2}{*}{ épaisseur (m) } \\
\hline & & & \\
\hline $\begin{array}{l}\text { Valanginien } \\
\text { (marno-calcaires) }\end{array}$ & 43000 & 1800 & 3 \\
\hline $\begin{array}{l}\text { Portlandien } \\
\text { karstique }\end{array}$ & $\begin{array}{c}25000 \mathrm{a} \\
37000\end{array}$ & 1900 & 3 \\
\hline $\begin{array}{l}\text { Kimméridgien } \\
\text { inférieur }\end{array}$ & 38000 & 2500 & 2 \\
\hline Oxfordien & 25000 & 27000 & 2,8 \\
\hline
\end{tabular}

\subsection{La galerie de reconnaissance et le tunnel de} l’Épine

La galerie de reconnaissance a fait l'objet de nombreuses observations et mesures et peut encore être examinée avec intérêt car elle n'est que très partiellement revêtue, contrairement au tunnel autoroutier qui a été creusé beaucoup plus rapidement et revêtu sur toute sa longueur de béton coffré en voûte et de béton projeté ou coffré en piédroits (fig. 9).

La stabilité des roches a été assez bonne tant en galerie que dans le tunnel. Les instabilités ayant nécessité un soutènement par cintres se sont manifestées dans la dolomie poreuse ou microfissurée, dans les zones marneuses de l'Hauterivien, les karsts du Barrémien et les marnes du Chattien. La molasse Burdigalienne constituée de grès tendres présentait une tendance au délitage en plaques épaisses et a nécessité un boulonnage avec grillages ou plaques pour la sécurité du chantier dans l'attente du revêtement de béton. Le phénomène le plus remarquable est la décompression liée aux fortes contraintes dans la zone centrale comprimée. Un écaillage progressif des reins puis des naissances et du sommet des piédroits a été observé dans la galerie. Ce phénomène s'est lentement étendu jusqu'à régner entre les points métriques 900 et 1000 ; la zone intéressée correspond exactement à la partie centrale de la structure du massif. Le phénomène n'a pas été gênant dans le tunnel car il était suffisamment lent pour être neutralisé par la pose du revêtement. 


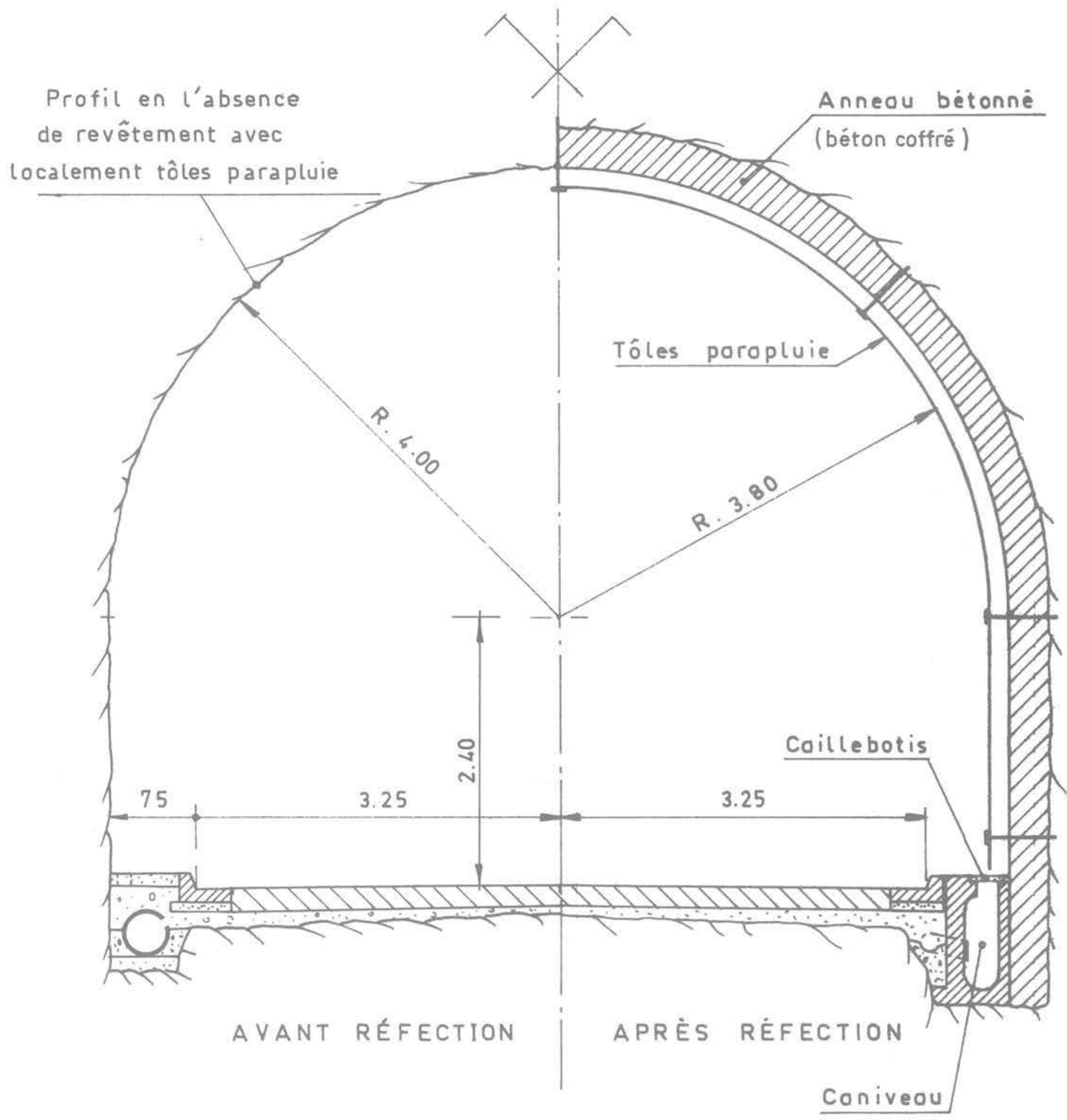

Fig. 8 Tunnel du Chat. Profil en travers 
SOUTE்NEMENT PAR CINTRES

REVÊTEMENT BÉTON COFFRÉ
PAS DE SOUTÈNEMENT

REVÊTEMENT BÉTON PROJETÉ

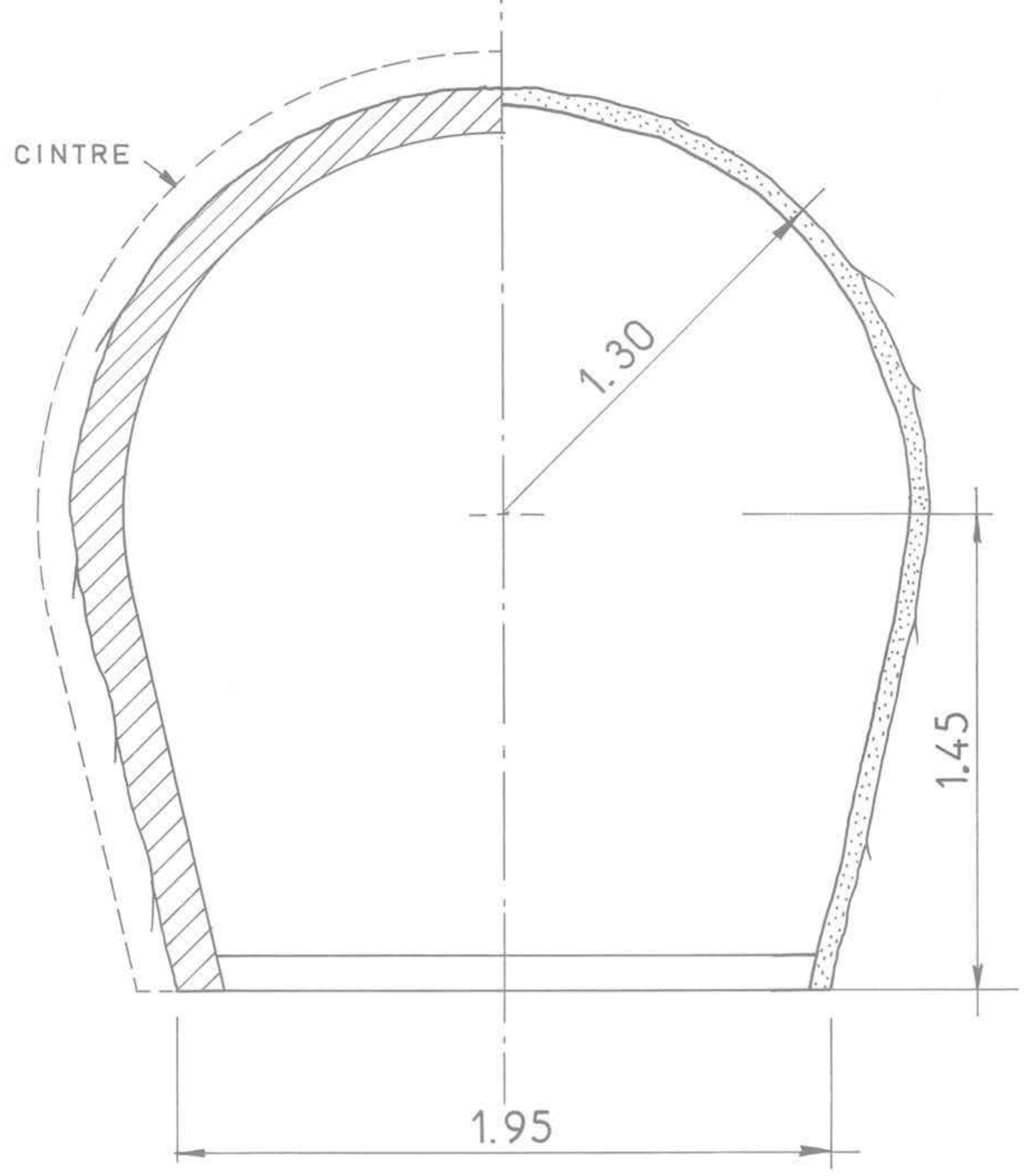

Fig. 9 Tunnel de l'Epine. Profil en travers 


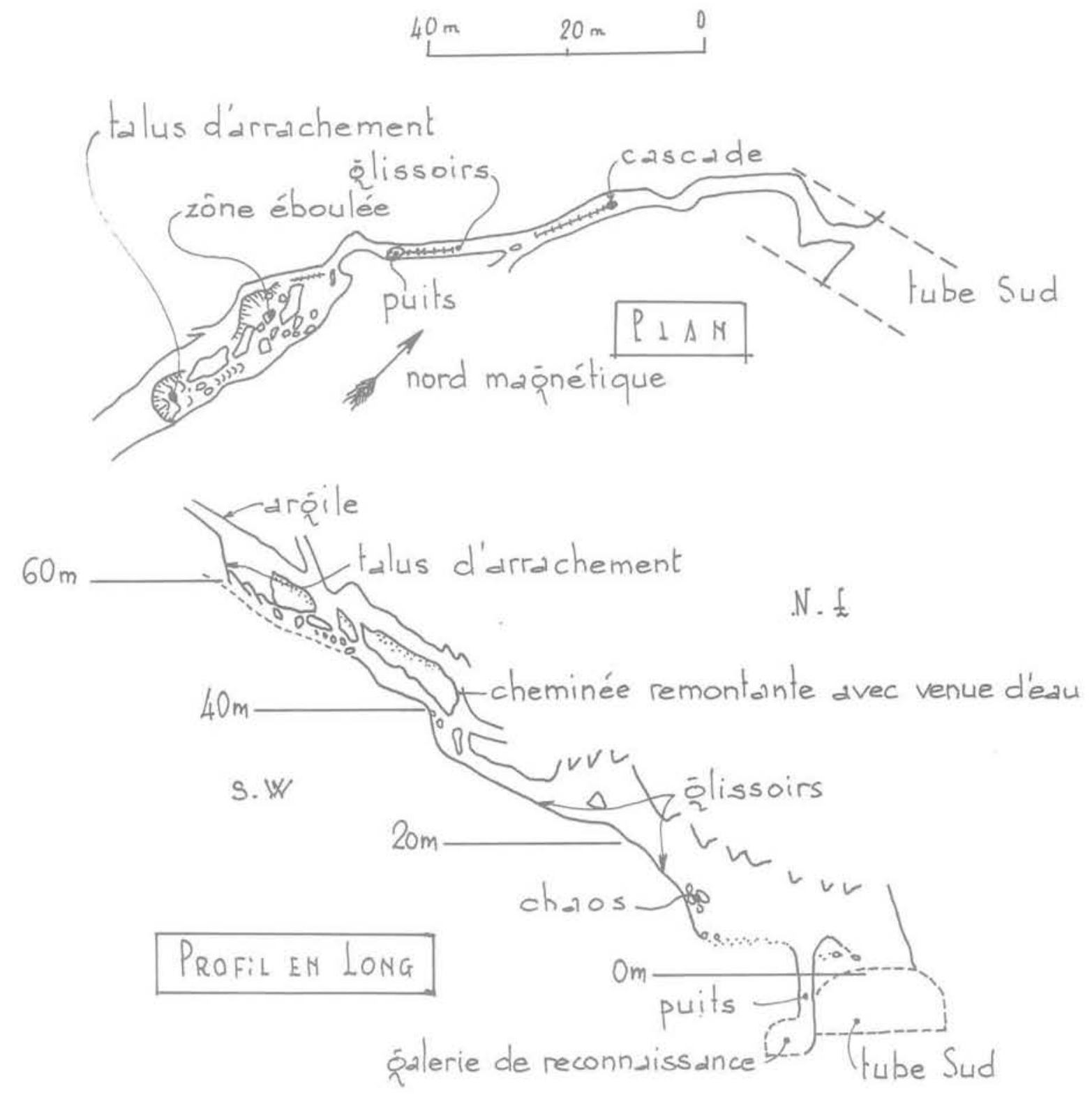

Fig. 10 Tunnel de l'Epine. Karst du Barrémien au pm 2570 env. 


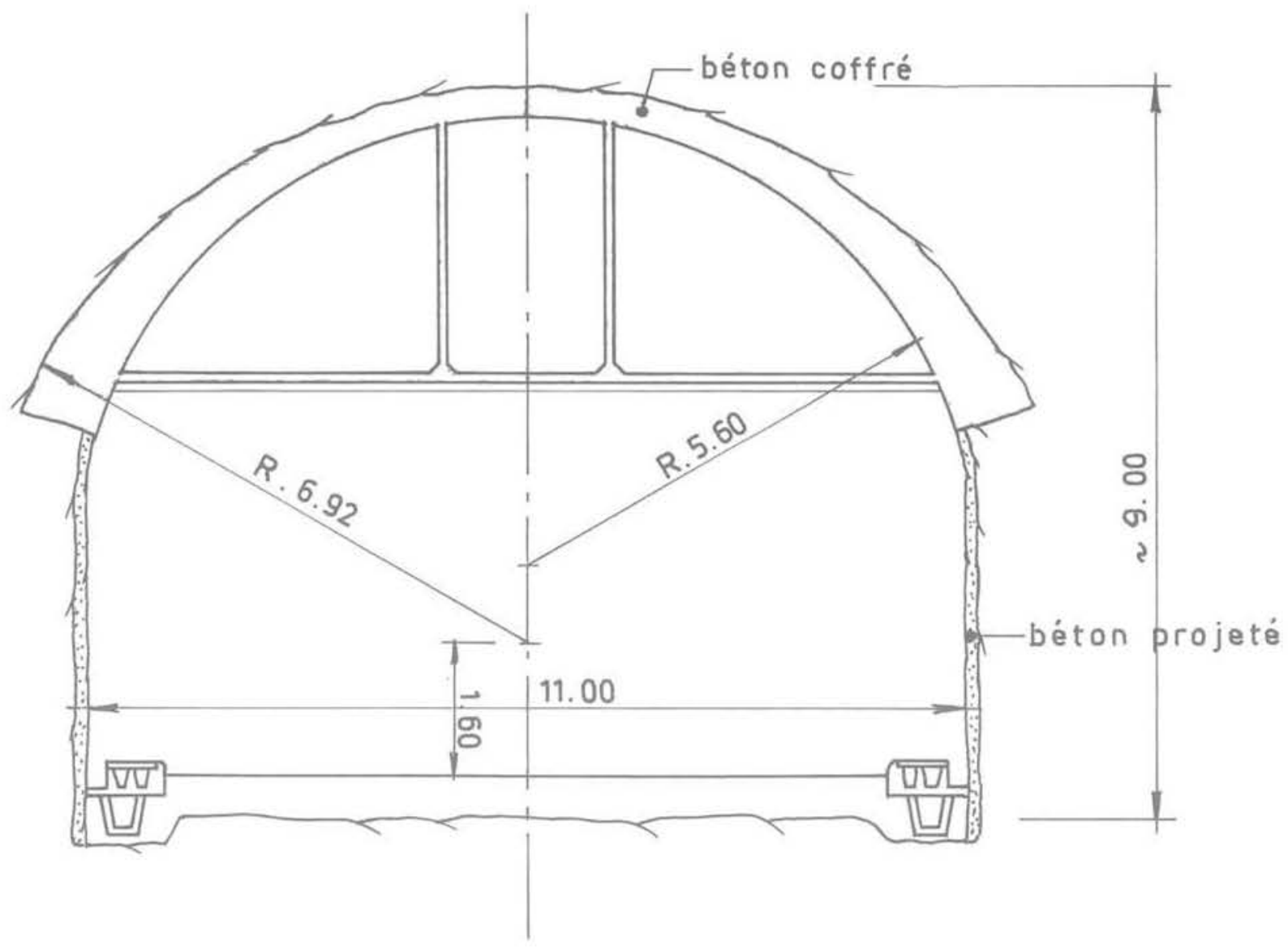

Fig. 11 Galerie du Bourget. Profil en travers

Les venues d'eau se répartissent irrégulièrement dans l'ouvrage. Dans la galerie de reconnaissance elles sont très abondantes et plutôt ponctuelles dans les calcaires des zones en tension aux extrémités de l'ouvrage: elles deviennent diffuses vers l'intérieur du massif sauf au droit de la zone dolomitisée. La zone centrale en compression est sèche. Les venues d'eau sont faibles dans les terrains tertiaires argileux à I'Est. Les débits de crue atteignent $2 \mathrm{~m}^{3} / \mathrm{s}$ dans la galerie de reconnaissance. La galerie joue bien un véritable rôle de drain. L'explication en est à rechercher dans la position de l'ouvrage dans la chaîne : à l'Est les calcaires sont recouverts par le Miocène imperméable au droit du tunnel, et en contre-bas, d'où mise en charge des zones de circulation - à l'Ouest, la galerie est située à $15 \mathrm{~m}$ seulement au-dessus de la cote du réseau hydrographique local (marais de Nances et Lac d'Aiguebelette). II en résulte que, à chaque précipitation importante, les eaux qui circulent rapidement dans le massif fissuré et karstique se mettent en charge dans les conduits d'écoulement au voisinage de la galerie qui devient un drain. Ce fait est confirmé par les nombreuses venues d'eau observables en base des piédroits en période de forte pluviosité.

Les karsts étaient déjà connus dans la montagne de l'Épine avant ces travaux - grande doline au sommet, source vauclusienne de la Conche au Nord. Des karsts actifs ont été rencontrés en souterrain au droit des accidents tectoniques (pm.1900) des zones dolomitiques (pm. 1 400). Le Barrémien a été intensément karstifié pendant la période d'émersion antetertiaire : il a été révélé un karst très important au pm. 2570 environ (fig. 10), mais dont s'est échappée une quantité notable de matériaux meubles. Des réseaux karstiques colmatés ont également été observés dans le Kimmeridgien et le Valanginien.

\subsection{La galerie du Bourget}

Les problèmes liés aux formations quaternaires (injections. congélation) ne seront pas développés dans ce paragraphe, consacré uniquement au comportement des roches secondaires et tertiaires.

La stabilité de l'excavation a été satisfaisante dans l'ensemble. Cependant, un écaillage des parois est apparu nettement dans la zone du cœur de I'anticlinal qui correspond également à la zone de plus forte couverture. Le phénomène s'est manifesté de la manière la plus caractéristique dans les roches les moins résistantes: marnes de l'Oxfordien et marno-calcaires du Bathonien. La forme élancée de la galerie (fig. 11) a favorisé l'écaillage en naissances et en piédroits. Par rapport à la galerie de I'Épine, la décompression s'est manifestée plus rapidement et son extension est plus grande, ce qui peut s'expliquer par la tectonique, mais certainement par l'épaisseur double du recouvrement. La zone neutre semble intéresser à l'Est l'Oxfordien supérieur et le Kimmeridgien inférieur (zone située entre la décompression et les karsts sur la figure 7): elle est beaucoup moins nette à l'Ouest.

Un soutènement par cintres métalliques a été nécessaire à l'Est dans certaines zones de calcaires karstiques et localement à l'Ouest dans des calcaires fissurés du compartiment proche des accidents tectoniques. Les formations conglomératiques de la base du Burdigalien ont également été cintrées à l'Ouest.

Les venues d'eau se manifestent essentielleraent, comme pour les autres galeries, dans les calcaires francs et dans la zone externe en tension. Le phénomène est net à l'Est oủ la structure est régulière : les calcaires sont aquifères depuis le Kimmeridgien supérieur au Barrémien à l'exception de 
I'Hauterivien marneux Quelques venues d'eau sont à noter également dans le compartiment Ouest où elles semblent liées aux accidents tectoniques. La zone centrale comprimée est pratiquement sèche.

L'ensemble des débits est de l'ordre de $150 \mathrm{l} / \mathrm{s}$. Par comparaison avec la galerie de l'Épine, on aurait pu s'attendre à des débits beaucoup plus importants, et ce d'autant plus que l'ensemble de l'ouvrage est situé à une côte inférieure à celle du réseau hydrographique voisin. Deux explications de ces débits modérés peuvent être avancées : la zone comprimée est assez étendue (de l'ordre du kilomètre), et les karsts sont colmatés (cf. ci-après) à la cote de la galerie. II est également probable que la structure du flanc Ouest n'est pas très favorable aux infiltrations et présente également des zones comprimées secondaires à perméabilité limitée.

Dans le cadre des contrôles effectués à l'avancement, la température et la résistivité de l'eau avaient été mesurées régulièrement. La température pratiquement constante depuis la tête s'est élevée progressivement de $13^{\circ}$ à $18^{\circ}$ pendant la traversée de l'Oxfordien. La résistivité, par contre, s'est abaissée brutalement dans l'Oxfordien supérieur de 35 à $100 \Omega \mathrm{m}$. Ces mesures montrent bien la transition entre deux régimes hydrogéologiques différents : eaux peu minéralisées circulant rapidement dans les calcaires francs de la zone en tension et eaux à circulation lente réchauffées et minéralisées dans la zone comprimée.

Les karsts se sont bien développés dans les calcaires francs du flanc Est comme aux tunnels du Chat et de l'Épine, mais ils sont pour la plupart colmatés de matériaux argileux ou argilo-sableux. Ce colmatage est à mettre en relation avec la cote beaucoup plus basse de cette galerie par rapport à celle des autres ouvrages. Les principales venues d'eau sont cependant apparues au droit de ces karsts, mais avec des débits relativement faibles, notamment par rapport à I'Épine. Certaines venues d'eau étaient alternativement claires, plus chargées en sable fin et à débit variable, témoignant de débourrages dans les conduits karstiques. II semble que la galerie soit située à une cote voisine du niveau de base actuel des karsts, et même peut-être légèrement en dessous, et qu'elle n'ait été que localement et partiellement alimentée par le réseau actif actuel.

\section{Conclusions}

Les études et les observations effectuées à l'occasion des travaux concernant les trois ouvrages souterrains du Chat, du Bourget et de l'Épine, situés dans le même chaînon jurassien du Chat et de l'Épine, permettent de dégager un certain nombre de conclusions concernant la structure du massif, les caractéristiques et le comportement des différentes formations géologiques, la méthodologie des études et du suivi des travaux

La structure du chaînon du Chat et de l'Épine, telle qu'elle apparaît à la faveur de ces trois galeries, met en évidence la formation d'un anticlinal ante-miocène à flanc Est régulier à pendage modéré, affecté systématiquement d'accidents tectoniques à l'Ouest. L'hypothèse d'accidents subhorizontaux assimilables à un chevauchement doit être retenue. En tout état de cause, ces accidents tectoniques se révèlent tous cassants et francs, sans broyages importants ni étirements de couches. Une voie à explorer pour préciser l'ensemble de la structure semble devoir être une étude microtectonique complète de surface et des trois ouvrages souterrains: la structure exacte au droit de la galerie du Bourget pourrait ainsi être précisée, et la détermination des mécanismes de formation de l'ensemble de la chaîne mieux appréhendés.

Les différentes formations géologiques s'avèrent de nature et d'épaisseur comparables au droit des trois galeries. Leur comportement mécanique présente également de grandes similitudes: I'Oxfordien et le Kimmeridgien inférieur sont toujours situés dans la zone centrale comprimée et présentent de nombreux plissements secondaires et plissotements. Des phénomènes d'écaillage dus aux fortes contraintes les affectent sous des couvertures supérieures à $600 \mathrm{~m}$. Les calcaires, très résistants pour la plupart, du Kimmeridgien moyen au Barrémien, sont de bonne tenue en souterrain sauf à proximité d'accidents tectoniques ou s'ils sont le siège d'une karstification intense. Ils sont généralement sitúés dans la zone en extension de l'anticlinal et affectés de fissures ouvertes colmatées ultérieurement de calcite ou d'argile qui ont favorisé le développement des phénomènes karstiques.

Les venues d'eau sont intimement liées à la nature lithologique des roches, à leur position dans l'anticlinal - dans la zone en compression ou dans la zone en tension - et à la position de l'ouvrage par rapport à la cote du réseau hydrographique et du manteau imperméable tertiaire ou quaternaire de la chaîne. Oxfordien et Kimmeridgien inférieurs sont secs. Les autres calcaires sont le siège de venues d'eau faibles dans la zone en compression, et peuvent donner lieu à des venues d'eau nombreuses et à fort débit dans la zone tendue de l'anticlinal si les conditions sont réunies pour que la galerie joue le rôle de drain.

Le suivi géologique des galeries pendant le creusement apparaît indispensable à la lumière de l'expérience de la galerie du Bourget où ont été utilisés tous les moyens à la disposition du géologue sur chantier et au laboratoire pour répondre, le mieux possible, aux préoccupations du Maître d'ceuvre et de l'Entrepreneur qui demandent à tout moment des renseignements précis pour prévoir et exécuter aux meilleures conditions de coot, de délai et de sécurité. II a fallu actualiser la connaissance générale de la région, collecter et interpréter constamment les observations et mesures faites en galerie, s'appuyer sur des examens micropaléontologiques et sédimentologiques pour élaborer en permanence de nouveaux schémas plausibles de structure. Malgré ces dispositions, il n'a pas toujours été possible de répondre avec toute la précision souhaitée aux préoccupations des responsables du chantier. Cependant, les dispositifs d'alerte mis en place devaient permettre d'éviter tout accident grave, et le Maître d'œuvre et l'Entrepreneur ont pu être avertis en temps utile de la forte probabilité, qui s'est confirmée, de l'allongement important de la zone de molasse à traverser à l'Ouest.

\section{Références Bibliographiques}

[1] BERNEDE J., HABIB P., PANET M. et PLOUVIEZ P. Mesures des contraintes naturelles dans la montagne de l'Épine. Deuxième Congrès de la Société Internationale de Mécanique des Roches - Communication 4.35 - 7 p. Belgrade, 1970.

[2] GIDON P. - Géologie Chambérienne. - Annales du Centre d'Enseignement Supérieur de Chambéry, 1963

[3] GUIGON F. - Géologie et travaux souterrains - Étude d'avant projet du tunnel du Bourget - Bilan des observations sur cinquante tunnels ferroviaires dans la région Rhône Alpes. Thèse de $3^{\circ}$ cycle. Grenoble, 1976.

14] LAMIRAUX C. - Géologie du miocène des chainons Jurassiens méridionaux et du Bas Dauphiné nord oriental entre Chambéry et La Tour du Pin. Thèse de $3^{\circ}$ cycle. Grenoble, 1977.

[5] WEBER P., PANET M. - Découpage et prédécoupage à l'explosif - Aspects théoriques aspects pratiques. Journées 1970 de Mécanique des Roches - Cahier 3 du Comité Français de Mécanique des Roches - Revue de I'Industrie Minérale pp. 89-98. (Numéro spécial du 15 juillet 1971). 
Intervention à la fin de la conférence de M. Darcy au Comité Français de Géologie de I'Ingénieur - École des Mines - Paris le 9 février 1978

\section{Pierre Antoine (IRIGM Grenoble)}

Je voudrais apporter quelques compléments d'information qui paraissent indispensables aux personnes qui ont étudié la géologie du projet de tunnel du Bourget-du-Lac et susceptibles d'intéresser, par leur caractère méthodologique, le public de géologues de cette conférence.

Je rappellerai en premier lieu que le choix de la solution adoptée (et en cours de réalisation) pour l'assainissement du lac du Bourget a résulté d'un concours d'idées auquel ont participé une cinquantaine d'entreprises et de bureaux d'études. Certaines des solutions proposées ne pouvaient l'être valablement sans une étude géologique, même sommaire. Ce fut notamment le cas pour la galerie entre le Bourget-du-Lac et le Rhône dont il est question ici. L'étude préliminaire fut demandée par un groupement d'entreprises au professeur R. Barbier de I'Université de Grenoble qui nous en chargea. Cette solution ayant remporté le concours il fallut approfondir notablement les recherches géologiques pour aboutir à une coupe géologique prévisionnelle correcte avant travaux et amasser toute information géologique de détail susceptible de faciliter le suivi des travaux. Ce long préambule a pour but de bien situer le contexte de l'étude, totalement différent de celui du tunnel autoroutier qui sert de référence. Dans notre cas l'étude fut commandée et financée par le groupement d'entreprises qui n'y consacra que des moyens très réduits et dont parfois les représentants intervinrent sans nous consulter dans le déroulement des rares travaux de reconnaissance entrainant ainsi, dans un cas au moins, la perte d'informations primordiales. Le sujet était néanmoins assez vaste et digne d'intérêt pour qu'un de nos élèves, M. F. Guigon ici présent, puisse en faire le sujet de sa thèse de $1 / 1^{\mathrm{e}}$ cycle soutenue le 29/06/75. Dans notre esprit ce travail devait revêtir deux aspects :

- essayer tout d'abord, dans un contexte favorable, de faire une prévision aussi précise que possible en utilisant toutes les ressources de la géologie de terrain. Cela nous était évidemment facilité par l'existence de tunnels précédemment forés dans le chainon et dont $M$. Darcy vient de nous remémorer les coupes géologiques; cela pouvait compenser, dans une certaine mesure, notre manque de moyens de reconnaissance. Le but était de montrer qu'une étude de terrain classique, menée consciencieusement par un géologue entraîné pouvait être efficace et valoriser beaucoup les indispensables reconnaissances complémentaires en permettant de les utiliser à bon escient:

- ensuite, par un suivi constant des travaux, passer au crible les résultats du travail précédent et notamment analyser en détail les éventuelles causes d'échec dans les prévisions. Ce travail est en cours sous la responsabilité de géologues de l'Université de Grenoble et nous disposons d'un levé détaillé, à l'échelle du 1/1000 des tronçons déjà exécutés.

On pourra nous objecter que nous nous placions dans des conditions favorables ayant bénéficié d'un acquis non négligeable par les reconnaissances et chantiers exécutés antérieurement. Une telle remarque ne peut s'appliquer qu'aux $4 \mathrm{~km}$ amont de l'ouvrage qui est long de treize. Sur $9 \mathrm{~km}$ nous fâmes donc bien obligés de nous contenter des levés et observations de terrain.

Les résultats obtenus à ce jour sont intéressants. D'une façon générale et jusqu'à la rencontre de laccident chevauchant dont M. Darcy nous a entretenu, je considère personnellement qu'en ce qui concerne les longueurs des divers terrains traversés tant sous le Mont Tournier ou le synclinal de Novalaise, que dans la moitié amont du chainon de l'Épine, la qualité des prévisions fut correcte. Le niveau repere du Callovien par exemple, sous mille mètres de couverture fut rencontré à $150 \mathrm{~m}$ près; la précision fut bien meilleure sous couverture plus faible, notamment sous le Mont Tournier. Sur le plan géotechnique nous avons par contre nettement sous-estimé les difficultés liées à la traversée de la formation molassique.

Pour ce qui est de la grande fracture du versant Ouest l'incertitude régnait quant à son attitude exacte. Aucun des tunnels précédents ne l'ayant traversée, nous recherchâmes des indices structuraux de surface permettant de trahir une éventuelle inclinaison, mais cela sans succès. En fait l'accident rencontré est d'un type très particulier qui n'a rien à voir avec la classique structure en pli-faille considérée comme classique dans ces régions. II s'agit probablement de la déformation tardive d'une structure ancienne selon un schéma dont nous commencons à avoir une certaine habitude dans les chaines subalpines et qui rompt avec une certaine routine intellectuelle.

En conclusion je pense que, malgré ce que, vu de l'extérieur, on peut considérer comme une erreur de prévision inadmissible, propre à confirmer la méfiance des techniciens vis-à-vis de la géologie, l'actuelle galerie du Bourget est exemplaire. Elle montre bien ce que peut apporter une géologie de terrain consciencieuse (bons résultats sur les trois quarts du tunnel), mais en révèle dans le même temps les limites. Elle souligne également le róle que peut lou doit ?) jouer chez le géologue une profonde connaissance de la région ainsi que l'aptitude à s'évader des schémas trop classiques. Quoiquil en soit nous projetons de résumer dans une publication à venir, à caractère méthodologique, les multiples enseignements du travail entrepris par le Laboratoire de Géologie de I'Université de Grenoble à l'occasion de l'exécution de ce tunnel. 
